\title{
La delimitación del ámbito rural: una cuestión clave en los programas de desarrollo rural
}

\section{Delimitation of the rural area: a key issue in rural development programs}

\author{
José Sancho Comíns* y Daniel Reinoso Moreno**
}

\section{INTRODUCCIÓN}

La estructura del poblamiento ha sufrido notables cambios en las últimas décadas en los países industrializados. La aparición de la sociedad postindustrial ha estado acompañada de nuevos procesos de asentamiento de la población; la confirmación de grandes áreas metropolitanas y la difusión de la «urbanización» a gran parte del territorio, son dos de sus principales características. La concentración de la población, que se agudiza al iniciarse el despegue económico, alcanza un punto álgido y, después, decrece en la medida en que aumentan los niveles de desarrollo del país y el impacto urbanizador alcanza a una buena parte del territorio (Vinuesa y Vidal, 1991).

En los últimos decenios se han producido transformaciones importantes en nuestro mundo rural; eso es hoy un hecho incuestionable. Estas transformaciones han implicado modificaciones espaciales, funcionales y socioeconómicas. Como ya señalaba la Organización para la Cooperación y el Desarrollo Económico (OCDE), hace ahora más de veinte años, «las estructuras sociales y económicas de las regiones rurales de la OCDE han cambiado considerablemente, al mismo tiempo que se alteraba su composición demográfica» (OCDE, 1988).

\footnotetext{
*Departamento de Geografía, Universidad de Alcalá (jose.sancho@uah.es).

** Departamento de Geografía, Universidad de Alcalá (daniel.reinoso@uah.es).
} 
De igual modo, en apenas un siglo, se ha producido una transformación radical de la ciudad (Remy y Voyé, 1976), en donde la ciudad preindustrial, generalmente como espacio amurallado, da paso a la difusión del fenómeno urbano y de las redes de urbanización a gran parte del territorio (Roca, 2003).

Estos procesos de cambio rural y descentralización urbana implican para algunos autores una modificación sustancial del concepto «rural», debido en buena medida a la extensión a todo el territorio de lo urbano: «la extensión a las áreas rurales del modo de vida urbano está borrando algunas de las características tradicionales que hacían del campo sobre todo un modo de vida, identificado con el de la civilización o mundo campesinos» (Molinero y Alario, 1994). Estos procesos generan también un nuevo marco de relaciones urbano-rurales y un profundo cambio estructural de las comarcas donde se desarrollan, llegando a desdibujar, en muchos casos, las fronteras entre los espacios rurales y los urbanos. Las diferencias, hasta bien entrado el siglo Xx, parecían claras e inequívocas, con una correspondencia entre «rural»y «agrario» y una morfología urbana caracterizada por la construcción en altura y la alta densidad de población; sin embargo, en pleno afianzamiento de la sociedad postindustrial, los contrastes son cada vez más difusos y difíciles de establecer. G. Duby afirmaba para Francia, ya en 1984, que «con toda evidencia, la más antigua división entre el campo y la ciudad se borra bajo nuestros ojos y este hecho se revela como una de las más drásticas mutaciones que afectan a nuestra civilización» (Kayser, 1990).

La propia definición de espacio rural, concebida inicialmente por oposición a lo urbano, está en entredicho, y se buscan nuevas fórmulas que recojan la nueva estructura y funcionalidad de las comarcas rurales. El concepto «rural» ha pasado, en poco más de 20 años, de ser sinónimo de tosco o atrasado a tener un cariz más notable y hasta ser sinónimo de «calidad». Las viejas tradiciones, símbolos y objetos, que en los tiempos del éxodo poblacional desde las áreas rurales se desechaban como manifestación del subdesarrollo, son ahora recuperados.

La Comisión Europea afirma que «el mundo rural abarcaría aquellas zonas y regiones donde se llevan a cabo actividades diversas e incluiría los espacios naturales y cultivados, los pueblos, villas, ciudades pequeñas y centros regionales, así como las zonas rurales industrializadas de dichas regiones, pero la noción del mundo rural no implica únicamente la simple delimitación geográfica. Evoca todo un tejido económico y social, un conjunto de actividades de lo más diverso: agricultura, artesanía, pequeñas y medianas industrias, comercios y servicios. Sirve de amortiguador y espacio regenerador, por lo que resulta indispensable para el equilibrio ecológico al tiempo que se ha convertido en un lugar privilegiado de reposo y ocio» (Comisión Europea, 1988). La plu- 
riactividad de este espacio rural no es, sin embargo, una novedad hasta hace poco desconocida, más bien ha sido y es consustancial al medio rural desde siempre; lo novedoso es el marco en el que se sitúan estas nuevas funciones (Sancho, 2002). Entre éstas cabe destacar, en los países más industrializados, las funciones ambientales, culturales y de ocio, que siempre sustanciaron un flujo de relaciones entre el medio rural y los habitantes urbanos y que, en la actualidad, se han fortalecido hasta convertirse en decisivas para ambos.

No es propósito fácil, por tanto, delimitar el espacio rural; sobre todo si esta delimitación lleva consigo implicaciones operativas en relación a la aplicación a los Programas de Desarrollo Rural. Veamos brevemente algunas referencias teóricas que pueden ayudarnos a perfilar el concepto de ruralidad y, después, presentaremos los intentos de delimitación espacial de lo rural con vistas a la aplicación de acciones de desarrollo.

\section{PeRsPeCtiva CLÁSICA DE LA RURALIDAd y ENSAYOS RECIENTES PARA SU DEFINICIÓN}

El concepto de rural se ha entendido clásicamente como partícipe de cuatro características comunes: en primer lugar, una densidad de población baja, determinada históricamente por la capacidad de explotar los recursos cercanos; en segundo lugar, por la importancia de la actividad agraria, que si bien va perdiendo peso progresivamente en los países más industrializados con respecto a otros sectores, es indudable que aún sigue siendo el centro sobre el que se articula mayoritariamente la economía de las comarcas rurales; en tercer lugar, la fuerte relación entre los habitantes y el medio natural que los rodea, que determina su propia identidad, actividad económica, fiestas, materiales y formas de construcción, etc.; y en cuarto lugar, las fuertes relaciones sociales que se establecen entre sus habitantes, marcadas por pertenecer a una colectividad de reducido tamaño y relativamente estable, hasta la afección del impulso migratorio.

Este concepto de «rural» por oposición a «urbano» sustenta las teorías clásicas del enfoque dicotómico, basadas en la dualidad y contraposición entre lo rural frente a lo urbano. Estas teorías implican una concepción de la ruralidad como sistema social tradicional, caracterizado por el peso de las tradiciones y costumbres o la falta de espíritu emprendedor frente a la sociedad urbana moderna. Se trata, sin duda, de una visión superada por el nuevo perfil de la sociedad rural actual.

Frente al enfoque dicotómico, surge, ya en la primera mitad del siglo xx, la idea del «continuum rural-urbano». Sorokin y Zimmerman (1929) presentaron 
su teoría del «continuum», que supuso el punto de partida de numerosos estudios modernos. Para ellos, las diferencias entre sociedades rurales y urbanas son graduales, no existiendo un inequívoco punto de ruptura entre ambas y estableciendo, en todo caso, como variable generadora del gradiente la proporción de agricultores. En este mismo esquema conceptual del «continuum», Wirth (1938) presenta, sólo unos años más tarde, una teoría similar pero cuya variable determinadora del «continuum» es la densidad demográfica. A mediados de los años 40, R. Redfield propone, desde una visión antropológica, otro gradiente en base al concepto de «folk-society» como tipo ideal de sociedad pequeña, aislada, homogénea y con fuerte sentido de la solidaridad de grupo, como contraposición a la sociedad urbana culturalmente avanzada (García, 1991).

En los últimos decenios, se han multiplicado los esfuerzos, principalmente desde una óptica urbana, por integrar los cambios que se están produciendo en el sistema de asentamientos a la clásica delimitación urbano-rural, intentando remarcar las características diferenciadoras que aún existen. En este sentido, Roca (2003) aborda una revisión crítica de las principales metodologías de delimitación urbana, teniendo ya presente los modernos fenómenos de desconcentración urbana. Este autor concluye que ya no se puede hablar de una frontera entre lo urbano y lo rural sino de un «gradiente de urbanización», gradiente en la intensidad de edificación, en la actividad y en la estructura económica, en la movilidad de personas y de mercancías, etc.

Dicho lo anterior, el empeño por marcar una frontera geográfica entre lo rural y lo urbano sigue siendo tan difícil como necesario. De igual modo que no existe una división clara entre sociedad rural y urbana, tampoco hay un criterio de división territorial único aplicable a ámbitos geográficos de diferente condición. Las transformaciones rurales y la extensión de lo urbano, muy especialmente por el auge espectacular, en primer lugar, de los medios de transporte y, más tarde, de los medios de comunicación, han supuesto un acercamiento entre ambas sociedades, a la vez que dificultan la ya de por sí ardua tarea de dibujar una frontera territorial entre ambos espacios. En todo caso, parece claro que, como ya señaló P. George en 1969, es inútil buscar definiciones de alcance universal aplicables a ámbitos muy diferentes.

Sin embargo, la necesidad operativa por encontrar criterios de delimitación urbano/rural ha incentivado la investigación científica. Los resultados apuntan a dos tipos de soluciones metodológicas; por un lado, las que se basan en criterios cualitativos, como son el estatuto jurídico, la función administrativa, la existencia de determinados servicios, etc., y, por otro, las que se apoyan en criterios cuantificables, entre los que cabe destacar el número de habitantes, la densidad de población y la proporción de empleo agrario (Capel, 1975). 
Para la delimitación cuantificable se han desarrollado diferentes métodos basados en datos e índices estadísticos más o menos complejos; la mayoría de los métodos de clasificación discreta están basados en indicadores simples, que, si bien facilitan la clasificación, representan una notable simplificación de la realidad, en algunos casos se combinan distintos indicadores simples para paliar el empobrecimiento que conlleva su utilización. Los indicadores más utilizados son los siguientes:

- El tamaño demográfico: el número de habitantes es, sin duda, el método más utilizado en todo el mundo por los institutos de estadística y los organismos oficiales para la clasificación de un núcleo de población como rural o urbano. Se enfrenta a tres problemas fundamentales: en primer lugar, establecer el área administrativa a que se aplica; en segundo lugar, dos núcleos de igual número de habitantes no tienen por qué tener el mismo carácter rural; en tercer lugar, y común para todos los índices, resulta complejo establecer el umbral a partir del cual se fija la delimitación. Existe una gran disparidad de criterios y, como en los casos anteriores, es imposible establecer un límite de carácter universal. Cloke y Park (1985) realizan una minuciosa comparación de distintos países encontrando una gran disparidad que va desde los 1.000 a los 20.000 habitantes como umbral de lo urbano.

- La densidad de población: como complemento al anterior, es también de común uso como criterio delimitador; tiene como ventaja sobre el anterior que da una idea de la intensidad de ocupación de la población sobre el territorio, pero su representatividad está condicionada en muchos casos por la extensión del municipio.

- Un determinado tipo de actividad económica: un neto predominio de los usos extensivos del suelo es una característica innata del medio rural por contraposición al urbano. El porcentaje de población con ocupación agraria ha sido uno de los datos más utilizados y, si bien ha disminuido mucho este porcentaje en los países más industrializados, aún continua siendo una característica propia del medio rural.

En algún caso, aprovechando las cada vez más abundantes y fiables estadísticas, se han desarrollado métodos de discriminación basados en indicadores complejos. Se puede decir que existen tantos indicadores complejos como estudios sobre el tema, uno de los primeros y quizás el más conocido es el índice de ruralidad de Cloke (1977). Este autor combina 16 variables de los censos de Inglaterra y Gales de 1961 y 1971 y elabora una clasificación en 5 grandes grupos que van desde las áreas más aisladas con descenso y envejecimiento de 
la población a los espacios residenciales periurbanos. La tipología resultante cristaliza en cinco clases: rural extremo, rural intermedio, periurbano intermedio, periurbano y áreas urbanas. Camarero (1993) realiza un exhaustivo análisis de diferentes indicadores complejos concluyendo que:

«...estas técnicas pueden llegar a confundir más que a aclarar sobre el significado de ruralidad. En el fondo estos trabajos son profundamente deudores de la disponibilidad estadística de indicadores y terminan reflejando, en función de esa disponibilidad, los intereses administrativos de los productores estadísticos,... La enorme variedad de los indicadores utilizados llega a determinar una definición de ruralidad altamente generalizable, de lo que resulta de una validez limitada».

En España, al igual que en otros países industrializados, a partir de la década de los años 70 comienza a ser más difícil la diferenciación entre espacios rurales y urbanos, al desmerecer lo rural en sus características históricas y aumentar la influencia de las urbes en el ámbito rural. En nuestro país, han predominado los estudios de delimitación urbana frente a los de óptica rural. Uno de los pioneros y más interesantes es el de Díez Nicolás (1972); este autor realiza diferentes aproximaciones estadísticas para la delimitación de áreas urbanas con datos del Censo de Población de 1960 ${ }^{1}$.

Entre los escasos estudios centrados en el ámbito rural cabe destacar el trabajo de Julián Mora (1990 y 1991) que, mediante la técnica de análisis factorial de componentes principales, analiza los municipios de Extremadura. Para ello, tiene en cuenta 56 variables, agrupadas en 5 bloques -físico-natural, socio-económico, demográfico, nivel de vida y equipamientos e infraestructuras $^{2}$ - Basados también en el análisis de componentes principales se encuen-

${ }^{1}$ Concluye que la delimitación que se aproxima más a la realidad está establecida por tres parámetros. Partiendo de municipios de más de 10.000 habitantes, toma la entidad capital más las entidades en un radio de $5 \mathrm{~km}$, si el municipio tiene entre 10.000 y 50.000 habitantes, o un radio de $10 \mathrm{~km}$ si el municipio es de más de 50.000 habitantes; si la suma es mayor de 10.000 habitantes considera el municipio como urbano. Un segundo criterio es la actividad económica, el 50\% de actividad ha de ser no agrícola, incrementándose este límite hasta el $60 \%$ si el municipio tiene más de 20.000 habitantes. Como tercer criterio la densidad de población ha de ser mayor de $60 \mathrm{hab} / \mathrm{km}^{2}$.

2 Centrándose en los factores urbano y demográfico, que explican el 30\% de la varianza, llega a diferenciar cuatro niveles en los que se localizan los asentamientos: urbano, urbanizado, semirural y rural. También establece una tipología de asentamientos en función de la evolución reciente de su comportamiento demográfico: asentamientos muy dinámicos, dinámicos, menos dinámicos y regresivos. Por último, en función de las interrelaciones existentes entre el factor urbano y el demográfico configura un «modelo extremeño de asentamientos humanos» formado por 4 categorías: urbano en expansión, rural atrasado, urbano en regresión y rural desarrollado. 
tran los estudios del Instituto Nacional de Investigaciones Agrarias (INIA, 1983 y 1983), que llegan a delimitar el grado de depresión socioeconómica a nivel comarcal a partir de 20 variables del medio físico y socioeconómico, con un fuerte componente agrario.

En los últimos años la discusión científica sobre la delimitación urbanorural en España se ha centrado en la definición de lo rural desde una perspectiva demográfica y, en particular, en la utilización de la entidad de población o el municipio como unidad de medida y en el establecimiento de sus límites cuantitativos (García, 1994; Camarero, 1991 y 1993; Vinuesa 1997 y Vidal, 1989), siendo el municipio, como es bien sabido, una delimitación territorial administrativa y la Entidad Singular de Población, cualquier parte habitable del término municipal, habitada o excepcionalmente deshabitada, claramente diferenciada dentro del mismo y que es conocida por una denominación específica, según la propia definición del Instituto Nacional de Estadística.

La discusión no es baladí ya que, como se aprecia en la tabla 1, las diferencias entre lo que se computa como población rural ${ }^{3}$ utilizando el municipio o la entidad de población como unidad de medida son apreciables. A nivel nacional, 6,6 millones de habitantes son catalogados como rurales, de utilizar el criterio entidad de población, frente a los 2,8 millones en el caso de partir de los municipios como unidad de medida. Este doble resultado se debe a dos factores bien diferentes; por un lado, existen municipios cuya población total supera los 2.000 habitantes, por lo que no son considerados municipios rurales, si bien son una agregación de entidades que ninguna de ellas alcanza esta cifra de población; por otro, existen cada vez más entidades con población inferior a los 2.000 habitantes que se encuentran en municipios de gran población principalmente en las áreas metropolitanas y zonas de costa y, sin embargo, son consideradas rurales al contabilizar la población por entidades.

Las diferencias se hacen aún más notables al considerar la delimitación por provincias debido a los diferentes sistemas de asentamientos (Sancho y Reinoso, 2003), la utilización del municipio en lugar de la entidad de población produce distorsiones importantes en las provincias gallegas y en el Principado de Asturias y, en menor medida, en el resto de provincias de la cornisa cantábrica y Murcia, donde existe una gran dispersión de la población municipal en distintas entidades.

${ }^{3}$ El Instituto Nacional de Estadística utiliza la entidad singular de población para delimitar 3 tipos de áreas: Zona urbana, conjunto de entidades singulares de población con 10.001 o más habitantes; Zona intermedia, conjunto de entidades singulares de población de 2.001 a 10.000 habitantes; Zona rural, conjunto de entidades singulares de población con 2.000 o menos habitantes. 


\section{MAPA 1}

TIPOLOGÍA DE LOS MUNICIPIOS ESPAÑOLES (CRITERIO INE)

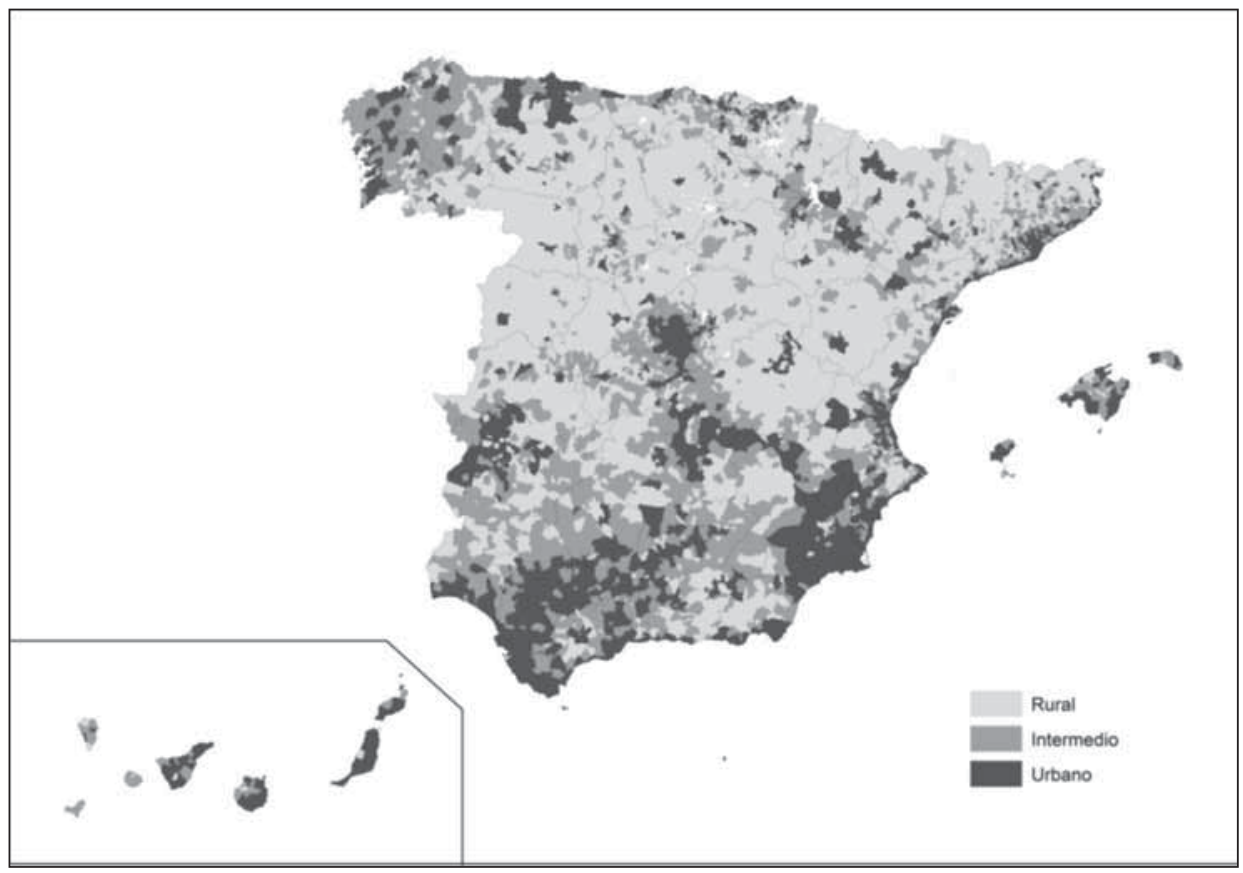

Fuente: elaboración propia a partir del Padrón Municipal de Habitantes (INE, 2010).

TABLA 1

TIPOLOGÍA DE MUNICIPIOS Y ENTIDADES SEGÚN EL VOLUMEN DE POBLACIÓN

\begin{tabular}{|c|c|c|c|c|c|c|c|c|}
\hline \multirow[b]{2}{*}{ TIPOLOGÍA } & \multicolumn{4}{|c|}{ MUNICIPIOS } & \multicolumn{4}{|c|}{ ENTIDADES } \\
\hline & Número & Población & $\begin{array}{l}\% \text { de } \\
\text { Muni. }\end{array}$ & $\begin{array}{l}\% \text { de } \\
\text { Pob. }\end{array}$ & Número & Población & $\begin{array}{l}\% \text { de } \\
\text { Entid. }\end{array}$ & $\begin{array}{l}\% \text { de } \\
\text { Pob. }\end{array}$ \\
\hline RURAL & 5.789 & 2.811 .766 & 71,3 & 6,0 & 58.984 & 6.660 .620 & 95,8 & 14,2 \\
\hline INTERMEDIO & 1.574 & 7.111 .922 & 19,4 & 15,1 & 1.910 & 8.351 .439 & 3,1 & 17,8 \\
\hline URBANO & 751 & 37.097 .343 & 9,3 & 78,9 & 666 & 32.008 .972 & 1,1 & 68,1 \\
\hline TOTAL & 8.114 & 47.021 .031 & 100 & 100 & 61.560 & 47.021 .031 & 100 & 100 \\
\hline
\end{tabular}

Fuente: elaboración propia a partir del Padrón Municipal de Habitantes (INE, 2010).

Estudios Geográficos, Vol. LXXIII, 273, pp. 599-624, julio-diciembre 2012

ISSN: 0014-1496, eISSN: 1988-8546, doi: 10.3989/estgeogr.201221 
A favor de la utilización del municipio cabe decir que se evita la consideración de núcleos rurales a las cada vez más numerosas urbanizaciones que se crean en los municipios cercanos a las grandes ciudades. Por último, a nivel de entidad, sólo se dispone de los datos de población total y su distribución por género, lo que no permite realizar un estudio en profundidad de las características estructurales de la población, siendo además el concepto de entidad vago y cambiante, por lo que la comparación de cifras de censos sucesivos es arriesgada (Vidal, 1989).

En el fondo, esta discusión no es más que la constatación de dos hechos ya comentados: por un lado, la diversidad del origen histórico de poblamiento y de su división administrativa y, por otro, la dificultad de establecer criterios de general aplicación para una delimitación de lo rural.

Se puede realizar una aproximación al montante total de la población rural en España añadiendo a los municipios rurales — con población menor de 2.001 habitantes-, aquellos municipios que si bien cuentan con una población superior a esta cifra, no tienen en su interior ninguna entidad de población que alcance dicho tamaño. De este modo evitaremos la consideración de urbano o intermedio a aquellos municipios que son una agregación de núcleos de pequeño tamaño. Esta nueva medida nos permite afinar algo más el concepto rural y su concreción recibe el nombre de «municipio rural ampliado».

TABLA 2

TIPOLOGÍA DE MUNICIPIOS APLICANDO EL CRITERIO «MUNICIPIO RURAL AMPLIADO»

\begin{tabular}{lrrrr}
\hline $\begin{array}{c}\text { TAMAÑO } \\
\text { (HABITANTES) }\end{array}$ & Número & Población & $\begin{array}{c}\% \text { de } \\
\text { Municipios }\end{array}$ & $\begin{array}{c}\% \text { de } \\
\text { Población }\end{array}$ \\
\hline RURAL & 6.127 & 4.023 .259 & 75,5 & 8,6 \\
INTERMEDIO & 1.374 & 7.947 .258 & 16,9 & 16,9 \\
URBANO & 613 & 35.050 .514 & 7,6 & 74,5 \\
TOTAL & 8.114 & 47.021 .031 & 100 & 100 \\
\hline
\end{tabular}

Fuente: elaboración propia a partir del Padrón Municipal de Habitantes (INE, 2010).

Según los datos del Padrón de Habitantes de 2010, existen 338 municipios con población superior a los 2.000 habitantes que no tienen ninguna entidad de población de ese tamaño y acogen a una población de 1.211.493 habitantes (tabla 2), estando casi la mitad de los mismos en las cuatro provincias galle- 
gas. Esta población, sumada a los 2,8 millones de habitantes de los municipios rurales, daría una población rural superior a los 4 millones de habitantes. Si realizamos la misma operación tomando el umbral de 10.000 habitantes, rural e intermedio, en el que hay 138 municipios que superan esta población sin tener ninguna entidad que la alcance, la población rural ascendería a casi 12 millones de habitantes.

\section{DELIMITACIÓN DE TERRITORIOS Y NÚClEOS RURALES POR PARTE DE LOS ORGANISMOS INTERNACIONALES}

Como ya se ha comentado, las características que diferencian las áreas urbanas de las rurales difieren de un país a otro. La distinción, por tanto, entre población rural y urbana no puede condensarse en una sola definición aplicable a todos los países, y ni siquiera a la mayoría de los países de una región o ámbito zonal/continental.

Diferentes centros estadísticos de organismos internacionales trabajan para recopilar y homogeneizar esta información con el fin de poder realizar estudios supranacionales. La Organización de Naciones Unidas genera, de forma periódica, información estadística de sus países miembros y realiza recomendaciones para la elaboración de los censos de población y vivienda; por otro lado, la Organización para la Cooperación y el Desarrollo Económico (OCDE) y el centro de estadísticas de la UE (EUROSTAT) proponen índices e indicadores únicos para sus ámbitos de actuación, con el fin de poder comparar y aplicar planes y políticas comunes.

\subsection{Naciones Unidas}

El departamento de estadística de las Naciones Unidas solo recomienda, con carácter general, la utilización de la localidad, o en su defecto la unidad mínima administrativa de cada país, como unidad de medida (ONU, 1988); para un ámbito más reducido, la Comisión Económica para Europa —dependiente de este organismo- - recomienda que para facilitar la comparación se definan como rurales aquellas localidades con una población inferior a los 2.000 habitantes (ONU, 1998).

Este mismo departamento de estadística de las Naciones Unidas realiza una recopilación anual de la delimitación urbano-rural que establece cada 
país (ONU, 2011). Las clasificaciones se pueden agrupar en 4 categorías dependiendo de los criterios utilizados en los 91 países de los que se dispone información:

- Cualitativa: es la más utilizada; la mitad de los países, 46 de los 91 países, se decanta por la utilización de criterios político-administrativos o de carácter histórico, estableciendo de forma oficial los que son núcleos urbanos. Es el caso de numerosos países de pequeño tamaño en los que sólo la capital es considerada urbana, y de otros países de África y Latinoamérica que establecen como urbano los centros administrativos (Egipto, Brasil, Tanzania o Ecuador).

- Cuantitativa Simple: un 28.5\% de los países de los que se dispone información (26 de los 91), realizan una clasificación en base, únicamente, al número de habitantes del núcleo de población, siendo más común en países de Norteamérica y Europa. La mayoría opta por la recomendación de la ONU estableciendo en 2.000 habitantes el umbral a partir del cual se considera urbano, si bien existen notables diferencias entre los 200 habitantes de Noruega o Groenlandia y los 10.000 de Portugal o Senegal.

- Cuantitativa Múltiple: 12 países, un 13.2\%, se decantan por la utilización conjunta de dos o más indicadores simples. En todos los casos se utiliza el número de habitantes, que puede combinarse con la densidad de población (Canadá), con el porcentaje de población activa agraria (Bostwana) u otras clasificaciones más complejas como en el caso de India o Japón.

- Cualitativa y Cuantitativa: unos pocos países, 7 de los 91, menos del 8\%, designan las áreas urbanas por una combinación de factores cualitativos y cuantitativos, donde la denominación de urbano se da con carácter oficial; pero, a diferencia de la clasificación cualitativa simple, priman a unos criterios estadísticos establecidos; es el caso de la Federación Rusa, Nicaragua o Sudan.

Los datos que ofrece Naciones Unidas permiten tener una visión global de la distribución de la población rural en el mundo. Como se aprecia en la tabla 3, existe una clara relación entre desarrollo y porcentaje de población rural. En los países desarrollados la población rural representa solo un $25 \%$ del total de habitantes, relación que se invierte en los países más desfavorecidos en los que tres de cada cuatro habitantes vive en comarcas consideradas rurales. 
TABLA 3

DISTRIBUCIÓN DE LA POBLACIÓN URBANA Y RURAL EN EL MUNDO (MILES)

\begin{tabular}{lrrrrr}
\hline \multicolumn{1}{c}{ Área } & $\begin{array}{c}\text { Población } \\
\text { Total }\end{array}$ & Urbana & Rural & $\begin{array}{c}\text { Urbana } \\
(\%)\end{array}$ & $\begin{array}{c}\text { Rural } \\
(\%)\end{array}$ \\
\hline MUNDO & 6.974 .036 & 3.632 .457 & 3.341 .579 & 52,1 & 47,9 \\
\hline Regiones más desarrolladas & 1.240 .380 & 964.240 & 276.140 & 77,7 & 22,3 \\
Regiones menos desarrolladas & 5.733 .657 & 2.668 .217 & 3.065 .439 & 46,5 & 53,5 \\
Los países menos desarrollados & 851.103 & 242.686 & 608.418 & 28,5 & 71,5 \\
\hline África & 1.045 .923 & 413.880 & 632.043 & 39,6 & 60,4 \\
Asia & 4.207 .448 & 1.895 .307 & 2.312 .140 & 45,0 & 55 \\
Europa & 739.299 & 539.010 & 200.289 & 72,9 & 27,1 \\
América Latina y el Caribe & 596.629 & 472.175 & 124.454 & 79,1 & 20,9 \\
América del Norte & 347.563 & 285.805 & 61.758 & 82,2 & 17,8 \\
Oceanía & 37.175 & 26.280 & 10.895 & 70,7 & 29,3 \\
\hline
\end{tabular}

Fuente: elaboración propia a partir de ONU, 2012.

La distribución por áreas geográficas escenifica lo que hemos indicado anteriormente. Entre Asia y África acogen a más del 88\% de la población rural del planeta. Países del África Subsahariana y de Asia Central muestran porcentajes de ruralidad que en muchos casos superan el $80 \%$ de la población total; en un estado intermedio, Centroamérica y algunos países de América del Sur, Norte de África y Europa del Este, mantienen unos porcentajes de población rural cercanos al 50\%; por último, Norteamérica, con una población rural inferior a uno de cada cinco habitantes, así como Europa y Oceanía, cuentan con los menores índices de población rural, siendo ésta cercana al 25\% de la población total.

\subsection{Organización para la Cooperación y el Desarrollo Económico (OCDE)}

El Programa de Desarrollo Rural de la OCDE establece un límite territorial de lo rural basado en la densidad de población y el número de habitantes del núcleo de mayor tamaño. La metodología se desarrolla en tres fases (OCDE, 1994) y es aplicable a regiones de la escala territorial 3 (TL3) de la OCDE, que en España se corresponde con las provincias:

- En primer lugar, se considera rural aquella comunidad local con una densidad inferior a 150 hab/ $\mathrm{km}^{2}$ (500 en el caso de Japón y Corea). 
- En segundo lugar, se establece una clasificación regional diferenciando tres tipos en función del porcentaje de población que vive en los municipios definidos anteriormente como rurales:

- «Región Predominantemente Rural»: regiones donde más del 50\% de la población vive en comunidades consideradas rurales.

- «Región intermedia»: cuando entre el 15 y el 50\% de la población es rural.

- «Región Predominantemente Urbana»: menos del 15\% de la población vive en comunidades rurales.

- Un tercer criterio puede cambiar una provincia de categoría en función del tamaño del mayor centro urbano que contenga:

- Una provincia clasificada como Predominantemente Rural pasa a Intermedia si contiene un núcleo urbano de más de 200.000 habitantes, y este contiene, al menos, el $25 \%$ de la población total.

- Una provincia clasificada como Intermedia pasa a Predominantemente Urbana si contiene un núcleo urbano de más de 500.000 habitantes, y este contiene, al menos, el $25 \%$ de la población total.

Aplicando la clasificación regional de la OCDE a sus países miembros se aprecia que el $40 \%$ de sus habitantes viven en regiones consideradas urbanas, que solo representan el 4\% del territorio, mientras que las regiones Predominantemente Rurales ocupan el $86 \%$ del territorio y sólo dan cabida a poco más de un cuarto de la población; por su parte, las regiones consideradas como Intermedias acogen al 32\% de la población en el 10\% del territorio (OCDE, 2002).

Dentro de esta generalidad pueden encontrarse, como es lógico, diferencias notables siempre que se desciende en la escala de trabajo. En algunos países, como la mayoría de los nórdicos, la población que vive en áreas predominantemente urbanas es minoritaria; en otros, como Bélgica, Reino Unido y Alemania, se da la situación contraria y cerca del $90 \%$ de la población se asienta en regiones urbanas; otros países se caracterizan por una estructura dual, en la que la mayoría de la población se asienta en ambos extremos -áreas predominantemente rurales y urbanas-, como es el caso de Irlanda o Portugal, mientras que en países como Francia o España predomina el asentamiento en regiones intermedias (OCDE, 2002).

Esta clasificación aplicada a España (tabla 4 y mapa 2), tomando como unidad de medida las provincias tal como recomienda la propia OCDE y la Dirección General de Agricultura de la UE, ofrece unos porcentajes de territorio considerado rural muy similares a las clasificaciones municipales vistas en el apartado anterior, si bien, el número de habitantes que acogen es significativa- 
TABLA 4

CLASIFICACIÓN DE LAS PROVINCIAS ESPAÑOLAS SEGÚN CRITERIO OCDE

\begin{tabular}{lrrrrr}
\hline \multicolumn{1}{c}{ TIPOLOGÍA OCDE } & Provincias* $^{*}$ & Población & $\begin{array}{c}\text { Superficie } \\
\left(\mathrm{Km}^{2}\right)\end{array}$ & $\begin{array}{c}\text { \% de } \\
\text { Pob. }\end{array}$ & $\begin{array}{c}\text { \% de } \\
\text { Sup. }\end{array}$ \\
\hline Predominantemente Rural & 15 & 5.312 .993 & 205.687 & 11,3 & 40,7 \\
Intermedia & 25 & 17.685 .355 & 220.509 & 37,6 & 43,6 \\
Predominantemente Urbana & $12\left(^{*}\right)$ & 24.022 .683 & 79.146 & 51,1 & 15,7 \\
TOTAL & $52\left(^{*}\right)$ & 47.021 .031 & 505.343 & 100 & 100 \\
\hline
\end{tabular}

Nota: (*) Incluye Ceuta y Melilla como Predominantemente Urbanos.

Fuente: elaboración propia a partir del Padrón Municipal de Habitantes (INE, 2010).

MAPA 2

TIPOLOGÍA DE LAS PROVINCIAS ESPAÑOLAS (CRITERIO OCDE)

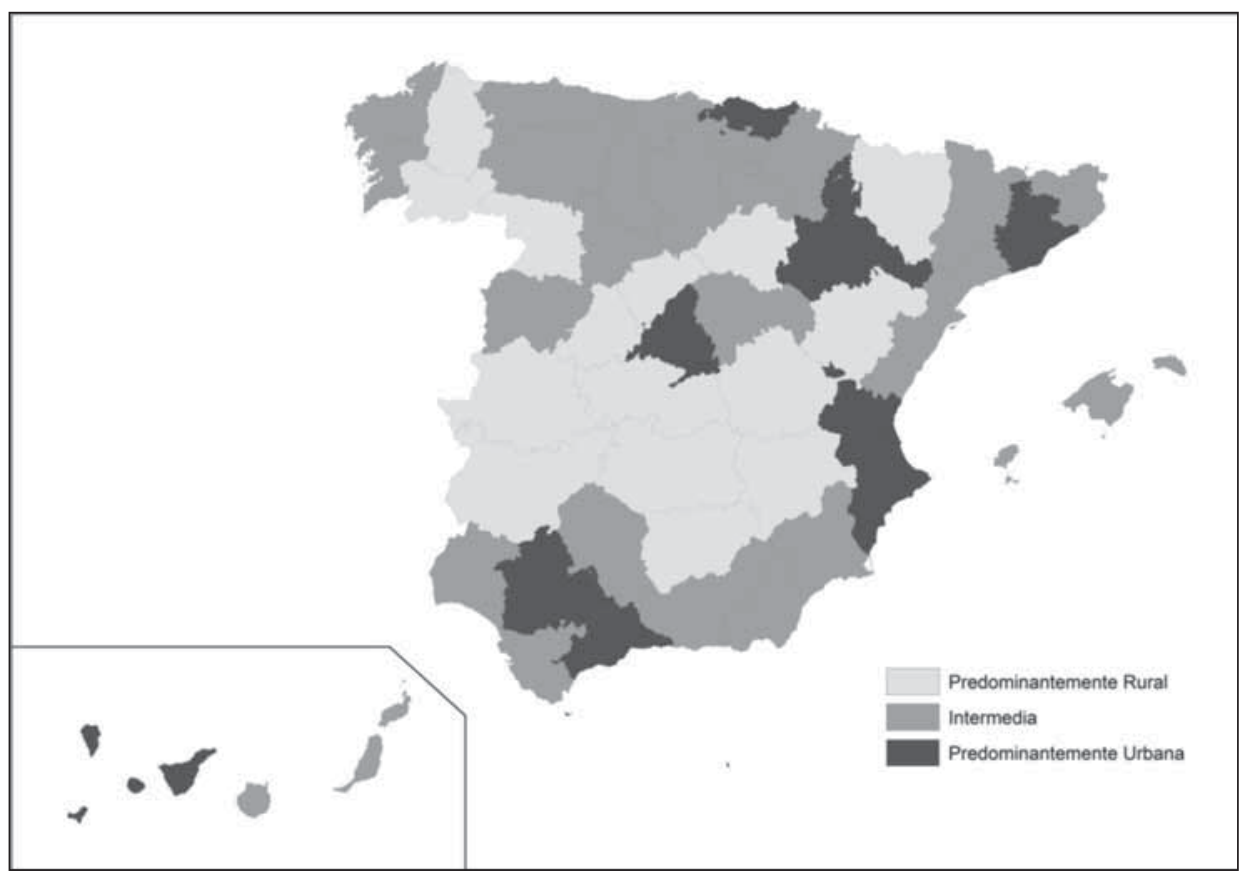

Fuente: elaboración propia a partir del Padrón Municipal de Habitantes (INE, 2010).

Estudios Geográficos, Vol. LXXIII, 273, pp. 599-624, julio-diciembre 2012 ISSN: 0014-1496, eISSN: 1988-8546, doi: 10.3989/estgeogr.201221 
mente mayor. Esto se debe a que en esta clasificación de la OCDE, con una zonificación supramunicipal, algunos municipios con población superior a los 2.000 o incluso a los 10.000 habitantes pueden quedar subsumidos en provincias en las que el peso de la población rural sea superior al 50\%.

Solo la aplicación del primer criterio de la metodología de la OCDE a los municipios españoles (mapa 3) ya da una primera idea de los problemas que pueden surgir; en primer lugar, porque el límite de densidad establecido, 150 $\mathrm{hab} / \mathrm{km}^{2}$, es extremadamente alto para España y, en segundo lugar, por la disparidad de tamaños que existe entre los términos municipales. Esto hace que de entre los 1.121 municipios clasificados como urbanos según este criterio, más de la mitad contengan menos de 10.000 habitantes, e incluso más de 100 no llegan a los 2.000 habitantes, mientras que entre los 6.993 clasificados como rurales encontremos más de 1.300 con más de 2.000 habitantes, e incluso 180 con más de 10.000 , entre ellos seis capitales de provincia.

MAPA 3

\section{MUNICIPIOS RURALES (DENSIDAD MENOR DE 150 HAB/KM²)}

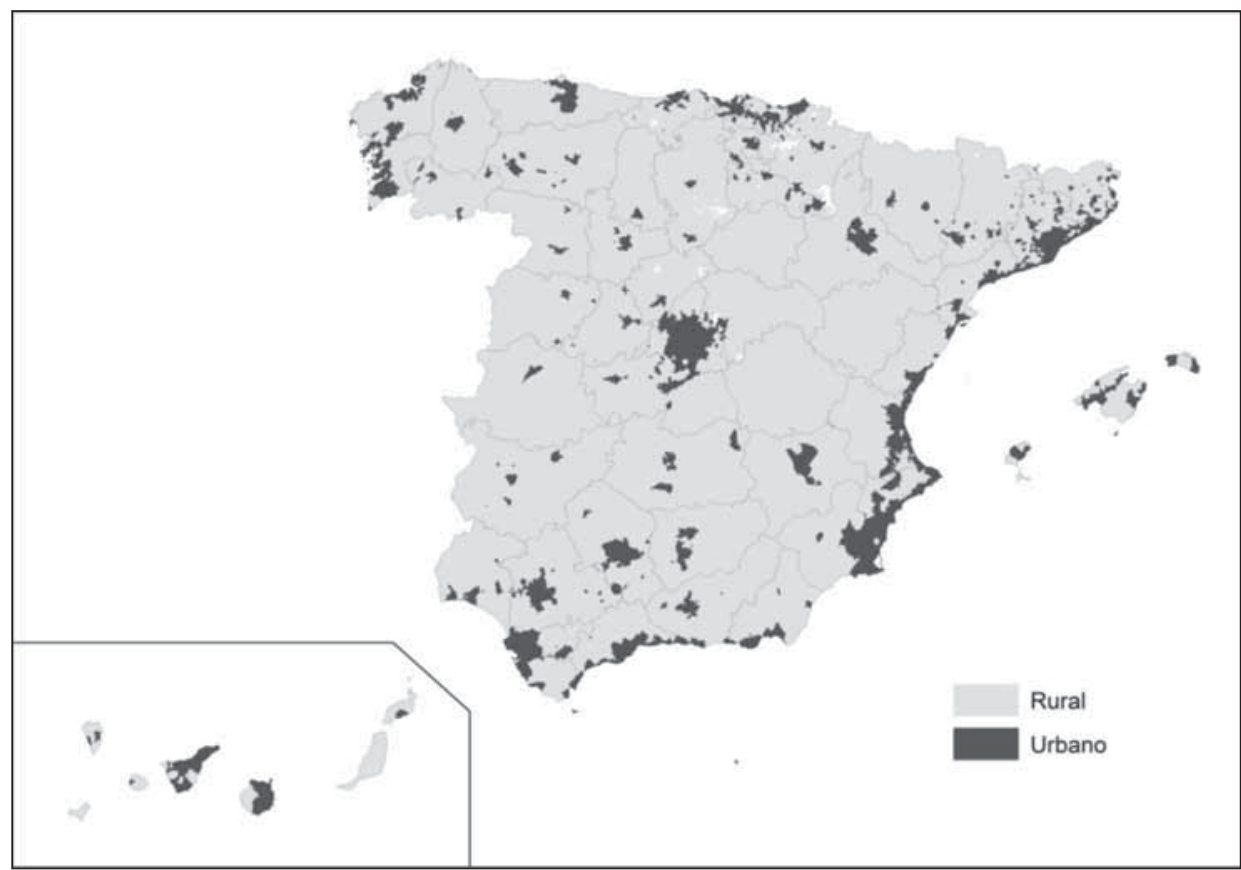

Fuente: elaboración propia a partir del Padrón Municipal de Habitantes (INE, 2010). 


\subsection{Agencia Estadística de la Unión Europea (EUROSTAT)}

La Agencia de Estadística de la Unión Europea (EUROSTAT), ha presentado recientemente una nueva metodología de clasificación urbano-rural (EUROSTAT, 2011) que es una adaptación de la metodología propuesta por la OCDE.

Esta nueva tipología pretende suplir uno de los mayores problemas vistos con anterioridad: la influencia que tiene la extensión superficial del municipio a la hora de calcular la densidad de población. Para ello, propone que la unidad territorial para la medida de la densidad sean cuadrículas de $1 \mathrm{~km}^{2}$, en vez de la utilización del término municipal.

Esta metodología utiliza dos criterios para cuantificar la población residente en zonas urbanas: uno relativo a la densidad y otro al tamaño demográfico mínimo. En primer lugar, las «celdas» del territorio de 1 kilómetro cuadrado han de tener una densidad de población superior a $300 \mathrm{hab} / \mathrm{km}^{2}$ para ser consideradas urbanas; en segundo lugar, el conjunto de celdas adyacentes que superen ese umbral de densidad han de tener una población mínima de 5.000 habitantes.

Para la delimitación a escala regional, la tipología de EUROSTAT aplica la metodología de la OCDE, con pequeñas variaciones, en regiones NUT3 — que en España se corresponde con las Provincias- a la vez que subraya el error metodológico de aplicar esta clasificación a regiones de mayor tamaño como las comunidades autónomas (NUT2). De esta forma, una provincia será considerada «predominantemente rural» cuando más del $50 \%$ de la población resida en las celdas consideradas no urbanas como consecuencia de la aplicación del criterio anteriormente citado, «intermedia», cuando entre el 20 y el $50 \%$ de la población se localiza en espacios rurales y «predominantemente urbana» cuando tenga menos del $20 \%$ de la población en territorio rural. Como en la metodología de la OCDE una provincia pasará de ser clasificada como «predominantemente rural a intermedia» si contiene un núcleo urbano de más de 200.000 habitantes, y una provincia clasificada como «intermedia» pasa a "predominantemente urbana» si contiene un núcleo urbano de más de 500.000 habitantes.

El resultado de la delimitación regional, en el caso de España, es muy similar al que se obtiene con la aplicación de la tipología original de la OCDE; así, utilizando el mismo año con el fin de hacer comparables los resultados sólo se producen dos cambios: Palencia pasa de ser considerada "predominantemente rural» en la metodología original a «intermedia», y la provincia de Guadalajara pasa de «intermedia» a «predominantemente rural». 
Esta nueva propuesta de EUROSTAR conviene tenerla muy en cuenta pues no sería extraño que en la nueva programación 2014-2020 de la política de desarrollo rural de la Unión Europea fuera decisiva a la hora de delimitar territorios susceptibles de acciones de desarrollo y, en suma, de recibir fondos que financien los mismos.

MAPA 4

TIPOLOGÍA DE LAS PROVINCIAS ESPAÑOLAS CON CRITERIO EUROSTAT

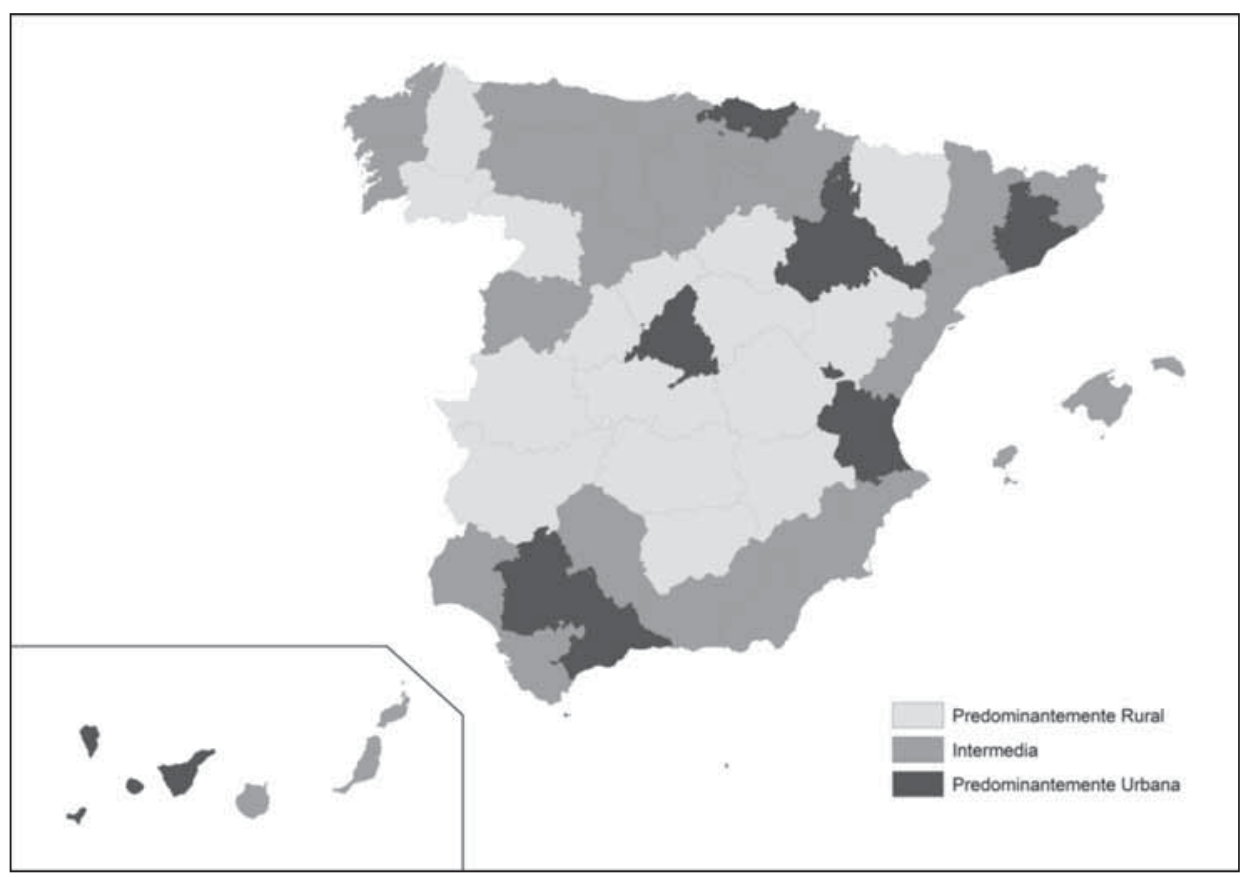

Fuente: EUROSTAT, 2011.

3. El ENFOQUe terRitorial EN LOS PROGRAMAS DE DESARROLlO RURAL EN EL MARCO DE LA UNIÓN EUROPEA

El tema tratado en este artículo - la delimitación de los territorios rurales- adquiere especial relevancia en los últimos veinte años dado el giro experimentado en la Política Agraria Común. De unos planteamientos de corte 
más agrarista se ha pasado a una mayor presencia del denominado «desarrollo rural» que ha tenido en la Iniciativa Comunitaria LEADER su más clara expresión. Esta, con un decidido enfoque territorial, ha demandado un esfuerzo en los gestores con el fin de delimitar su ámbito de actuación y hacer más coherentes sus acciones de desarrollo.

Así, para el periodo de programación en el que nos encontramos, 20072013, la Decisión del Consejo de 20 de febrero de 2006 sobre las directrices estratégicas comunitarias de desarrollo rural (2006/144/CE), recomienda la utilización de la definición de la OCDE para la delimitación del espacio rural, a escala NUT3, que en España corresponde a las provincias. En la misma se indica que si bien esta es «la única definición de zona rural reconocida internacionalmente, en algunos casos no tiene en cuenta plenamente la población que vive en zonas rurales... en el contexto de las presentes directrices se emplea exclusivamente a efectos estadísticos y descriptivos». En la misma línea, el Manual sobre el Marco Común de Seguimiento y Evaluación de las políticas de Desarrollo Rural 2007-2013, recomienda el uso del mismo indicador de ruralidad de la OCDE aplicado a escala NUT3, indicando, como en el caso anterior, que esta metodología puede no ser apropiada en todos los casos, y estipula que «cuando un estado miembro considere que la metodología de la OCDE no es apropiada, puede proponer y usar una delimitación alternativa».

Estas directrices europeas tienen su reflejo en el Plan Nacional de Desarrollo Rural, que marca las líneas fundamentales para la elaboración de los Programas de Desarrollo Rural de las Comunidades Autónomas. Este Plan, en su primera versión de 2007, afronta muy someramente el tema de la delimitación del espacio rural, limitándose a recoger la definición de la OCDE y a mostrar dos tablas, una con su aplicación a escala autonómica en la que se clasifica cada Comunidad en «Predominantemente Rural, Intermedia y Predominantemente Urbana», y otra a escala municipal, donde se recoge el porcentaje de territorio rural en cada caso. La segunda versión de dicho Plan (2009), ofrece, de forma más precisa, la identificación de las zonas rurales según el criterio de la OCDE aplicado a escala Autonómica y Provincial.

Con estos planteamientos iniciales, los criterios para la delimitación de las zonas rurales utilizados en los Programas de Desarrollo Rural por las Comunidades Autónomas son diversos, llegando, en algunos casos, a ser excesivamente complejos, y en muchos, a no estar suficientemente explicados los indicadores utilizados. 
TABLA 5

CRITERIO UTILIZADO PARA LA DELIMITACIÓN DE LAS ZONAS RURALES

\begin{tabular}{ll}
\hline \multicolumn{1}{c}{ CCAA } & $\begin{array}{c}\text { CRITERIO UTILIZADO } \\
\text { (Escala a la que se ha aplicado el criterio) }\end{array}$ \\
\hline Andalucía & OCDE (Toda la Comunidad) \\
Aragón & OCDE (Toda la Comunidad) \\
Asturias (Principado de) & OCDE (Municipal) \\
Balears (Illes) & OCDE (Municipal) \\
Canarias & OCDE (Municipal) \\
Cantabria & No especifica \\
Castilla y León & PROPIO (Municipal) \\
Castilla-La Mancha & PROPIO (Municipal) \\
Cataluña & OCDE (Municipal y comarcal) \\
Comunitat Valenciana & PROPIO (Municipal) \\
Extremadura & OCDE (Toda la Comunidad) \\
Galicia & OCDE (Municipal) \\
Madrid (Comunidad de) & OCDE (Municipal) \\
Murcia (Región de) & OCDE (Municipal) \\
Navarra (C. Foral de) & OCDE (Municipal) \\
País Vasco & PROPIO (Inframunicipal) \\
Rioja (La) & OCDE (Municipal) \\
\hline
\end{tabular}

Fuente: elaboración propia a partir de los Programas de Desarrollo Rural Autonómicos (ver bibliografía).

La mayoría de las Comunidades, 12 de 17, utilizan el criterio de la OCDE para la delimitación de las áreas rurales. De estas, tres comunidades - Andalucía, Aragón y Extremadura - aplican la metodología a escala autonómica, obteniendo como resultado la catalogación de «rural» para todo su territorio. Cataluña, combina el criterio de la OCDE aplicado a nivel municipal con una delimitación a escala comarcal basada también en la densidad de población, y el resto de este grupo de doce Comunidades Autónomas utiliza el límite de densidad recomendado de 150 habitantes por kilómetro cuadrado a escala municipal para la delimitación de las zonas rurales.

Entre las que no han adoptado la metodología propuesta por la OCDE las opciones son diversas. Castilla-La Mancha y la Comunidad Valenciana realizan una división a escala municipal sumando a la variable densidad demográ- 
fica otros indicadores. En el caso de Castilla-La Mancha, define tres tipos de espacios rurales: Zonas Rurales a Revitalizar, Zonas Rurales Intermedias y Zonas Rurales Semiurbanas, según a lo que especifica la Ley 45/2007 para el Desarrollo Sostenible del Medio Rural. La Comunidad Valenciana, por su parte, realiza una compleja clasificación municipal en función de la pirámide de edad, la densidad de población y los usos del suelo.

El País Vasco es la única Comunidad Autónoma que realiza una clasificación a escala inframunicipal, definiendo el espacio rural por oposición al espacio «urbano» teniendo en el paisaje un criterio decisivo al respecto. Mientras, Castilla y León realiza una delimitación en función de la población absoluta, considerando rural todos los municipios con menos de 10.000 habitantes. Por último, Cantabria no especifica ningún criterio estadístico, estableciendo como rural una «zona de referencia para las políticas de desarrollo rural que excluye las grandes poblaciones de la zona costera y sus áreas de influencia».

Cabe señalar que, aún utilizando el mismo criterio de clasificación, no todas las Comunidades son igual de concretas a la hora de delimitar el espacio rural, y las consecuencias que eso tiene en su Plan de Desarrollo, pudiendo dar lugar, en algunos casos, a indefiniciones o dudas sobre dónde y cómo se aplica el PDR. En este sentido, se pueden destacar como muy bien definidos los Planes de Navarra, Murcia, Madrid, Canarias o Cataluña.

La delimitación realizada en cada caso adquiere especial importancia en la aplicación del Eje 4 Leader en el marco de la nueva programación FEADER (Fondo Europeo Agrícola y de Desarrollo Rural), cuyas medidas solo afectan a los territorios regentados por los Grupos de Acción Local. Como se aprecia en la tabla 6, la mitad de las Comunidades Autónomas -Andalucía, Asturias, Baleares, Cantabria, Castilla y León, Comunidad Valenciana, Galicia, Madrid y Cataluña- establecen que el territorio en el que se puede aplicar el Eje 4 es el mismo que el definido como rural en cada caso. Otras tres Comunidades Canarias, Castilla-La Mancha y Navarra - amplían el área anteriormente definida como rural para la aplicación del Eje 4: Canarias con una delimitación inframunicipal en función de la altitud; Castilla-La Mancha a todos los municipios con menos de 30.000 habitantes, y Navarra a los municipios que distan más de $65 \mathrm{~km}$ del núcleo de Pamplona y a los enclavados en zonas desfavorecidas o de montaña, además de los definidos como rurales.

La Región de Murcia establece todo el territorio de su Comunidad como susceptible de recibir las medidas propias de la aplicación del Eje 4; de forma similar, en Extremadura es elegible toda su superficie menos 4 municipios, al igual que en La Rioja, que excluye 3 municipios. Aragón, por su parte, establece que tan solo queda excluida la misma zona que en la Iniciativa LEADER+. 
TABLA 6

ZONA DE APLICACIÓN DEL EJE 4

\begin{tabular}{|c|c|}
\hline CCAA & ZONA DE APLICACIÓN DEL EJE 4 \\
\hline Andalucía & Todo el territorio \\
\hline Aragón & Territorio LEADER + \\
\hline Asturias (Principado de) & Territorio definido como «rural» \\
\hline Balears (Illes) & Territorio definido como «rural» \\
\hline Canarias & $\begin{array}{l}\text { Territorio definido como «rural» matizado por } \\
\text { la altitud }\end{array}$ \\
\hline Cantabria & No especifica \\
\hline Castilla y León & Territorio definido como «rural» \\
\hline Castilla-La Mancha & $\begin{array}{l}\text { Todos los municipios de menos de } 30.000 \text { habi- } \\
\text { tantes }\end{array}$ \\
\hline Cataluña & Territorio definido como «rural» \\
\hline Comunitat Valenciana & Territorio definido como «rural» \\
\hline Extremadura & $\begin{array}{l}\text { Todo el territorio menos } 4 \text { Municipios (Cáce- } \\
\text { res, Plasencia, Badajoz y Mérida) }\end{array}$ \\
\hline Galicia & Territorio definido como «rural» \\
\hline Madrid (Comunidad de) & Territorio definido como «rural» \\
\hline Murcia (Región de) & Todo el territorio \\
\hline Navarra (C. Foral de) & $\begin{array}{l}\text { Territorio definido como «rural» además de los } \\
\text { que distan más de } 65 \mathrm{~km} \text { de Pamplona y los en- } \\
\text { clavados en zonas desfavorecidas o de montaña }\end{array}$ \\
\hline País Vasco & No define \\
\hline Rioja (La) & Todo el territorio menos 3 Municipios \\
\hline
\end{tabular}

Fuente: elaboración propia a partir de los Programas de Desarrollo Rural Autonómicos (ver bibliografía).

\section{CONCLUSIÓN}

La delimitación del ámbito rural, bien por intereses científicos u operativos, ha sido un tema ampliamente debatido. En la actualidad, si cabe, la preocupación es mayor, dada la repercusión que tiene a la hora de aplicar los Programas de Desarrollo Rural. En concreto, en España, los diecisiete Programas Autonó- 
micos toman opciones dispares para llevar a cabo esta exigencia postulada en las Directrices Estratégicas Comunitarias de Desarrollo Rural y en el propio Plan Nacional de Desarrollo Rural. Esta respuesta heterogénea, permitida desde las directrices citadas, encuentra una explicación en la diversidad del contexto geográfico y sociopolítico de España; no resulta siempre fácil dar una explicación coherente a las decisiones tomadas pues se mantienen en una franja difusa del acomodo a las propias condiciones territoriales de cada comunidad autónoma y la discrecionalidad sin aparentes razones fundadas.

Estas decisiones, no obstante, están influyendo decisivamente en la ejecución de los PDR. Uno de los postulados más sólidos de los mismos —el enfoque territorial - podría resentirse de no haber acertado e impactar de modo no deseable en esa cohesión que desde los PDR se busca para los territorios rurales. Sólo la evaluación «ex post» de los PDR podrá desvelar la bondad de cada una de las opciones y sonsacar un aprendizaje útil para el nuevo periodo de programación 2014-2020.

Recibido: 25/06/2012

Aceptado: 16/10/2012

\section{BiBLIOGRAFÍA}

Camarero, L. A. (1991): “Tendencias recientes y evolución de la población rural en España”. Política y sociedad, 8, pp. 13-24.

Camarero, L. A. (1993): Del éxodo rural y del éxodo urbano. Ocaso y renacimiento de los asentamientos rurales en España. Madrid, MAPA.

Capel, H. (1975): "La definición de lo urbano". Estudios Geográficos, 138-139, pp. 256-301.

Cloke, P. J. (1977): "An index of rurality for England and Wales". Regional Studies, II, pp. 143-152.

Cloke, P. J. y Park, C. (1985): Rural resource management. Londres, Croom Helm.

Comisión Europea (1988): El futuro del mundo rural. Madrid, MAPA.

Díez, J. (1972): Especialización funcional y Dominación en la España urbana. Madrid, Guadarrama.

EUROSTAT (2011): Eurostat regional yearbook 2010. Disponible en: http://epp.eurostat.ec.europa.eu/portal/page/portal/eurostat/home (Fecha de consulta: 26 de junio de 2012).

García, B. (1994): "Alcance y significado de las entidades singulares de población como concepto para cuantificar la población rural". Revista de estudios agrosociales, 168, pp. 199-234. 
García, J. M. (1991): "Sobre el concepto de ruralidad: crisis y renacimiento rural". Política y sociedad, 8, pp. 87-94.

George, P. (1969): Population et peuplement. Paris, Presses Universitaires De France.

INE (2010): Padrón de habitantes. Disponible en: http://www.ine.es/ (Fecha de consulta: 26 de junio de 2012).

INIA (1983): Análisis espacial de la depresión socioeconómica en España en base a las comarcas agrarias. Madrid, Mapa.

INIA (1983): Delimitación de zonas deprimidas en varias regiones españolas. Madrid, MAPA.

Kayser, B. (1990): La renaissance rurale: sociologie des campagnes du monde occidental. Paris, Armand Colin.

Molinero, F. y Alario, M. (1994): "La delimitación geográfica del desarrollo rural: una perspectiva historica". Revista de estudios agrosociales, 169, pp. 53-87.

Mora, J. (1991): "El modelo de asentamientos humanos en Extremadura". Estudios Territoriales, 36, pp. 129-145.

OCDE (1988): Nuevas tendencias en política rural. Madrid, ITUR, MOPU.

OCDE (1994): Creating rural indicators for shaping territorial policy. Paris, OCDE.

OCDE (2002): Territorial indicators of socio-economics patterns and dynamics. Paris, OCDE.

ONU (1988): Conceps and methods of environment statistics human settlements statistics a technical report. New York, ONU.

ONU (1998): Principios y recomendaciones para los censos de población y habitación. New York, ONU.

ONU (ed.) (2011): Demographic Yearbook 2009 - 2010. New York, ONU.

ONU (2012): Word Urbanization Prospects. The 2011 revision. New York, ONU.

Remy, J. y Voyé, L. (1976): La ciudad y la urbanización. Madrid, Instituto de Estudios de la Administración Local.

Roca, J. (2003): "La delimitación de la ciudad: ¿una cuestión imposible?". Ciudad y Territorio. Estudios Territoriales, XXXV/135, pp. 17-36.

Sancho, J. (ed.) (2002): Desarrollo rural. De los fundamentos a la aplicación. Madrid, Paraninfo.

Sancho, J. y Reinoso, D. (2003): "Población y poblamiento rural en España: un primer análisis a la luz del Censo de 2001". Serie Geográfica, 11, pp. 163-176.

Sorokin, P. A. y Zimmerman C. C. (1929): Principes of rural-urban sociology. New York, Henry Holt.

Vidal, T. (1989): La población rural de España. Cambios estructurales 1960-1980. Análisis del desarrollo de la población española en el periodo 1970 - 1986. Madrid, Síntesis, pp. 37-43.

Vinuesa, J. (1997): "El crecimiento de la población y los desequilibrios en la distribución espacial", en R. Puyol (ed.): Dinámica de la población en España. Madrid, Síntesis, pp. 265-310.

Vinuesa, J. y Vidal, M. J. (1991): Los procesos de urbanización. Madrid, Síntesis. 
Wirth, L. (1938): "Urbanism as a Way of Life". American Journal of Sociology, 44/1, pp. 3-24.

\section{Programas de Desarrollo Rural Autonómicos}

Programa de Desarrollo Rural de Galicia, 2007-2013. Xunta de Galicia, 2007. Disponible en: http://www.ceres.org.es/datos/comunidades/12/PDR_DE_GALICIA.pdf (Fecha de consulta: 04/09/2012).

Programa de Desarrollo Rural de la Comunidad Foral de Navarra, 2007-2013 (FEADER). Gobierno de Navarra, Departamento de Agricultura, Ganadería y Alimentación, 2007. Disponible en: http://www.redr.es/recursos/doc/Politicas_rurales/Programa_ desarrollo_rural/237423686_2112009175237.pdf y http://www.redr.es/recursos/ doc/ Politicas_rurales/Programa_desarrollo_rural/1495839666_2112009175341.pdf (Fecha de consulta: 04/09/2012).

Programa de Desarrollo Rural de la Comunidad Autónoma de La Rioja, 2007-2013. Comunidad Autónoma de La Rioja, Agricultura, ganadería y desarrollo rural, 2008. Disponible en: http://www.lariojasuroriental.com/programa/PDR_2007-2013.pdf (Fecha de consulta: 04/09/2012).

Programa de Desarrollo Rural de Aragón, 2007-2013. Gobierno de Aragón, Departamento de Agricultura y Alimentación, 2008. Disponible en: http://www.aragon.es/ estaticos/ImportFiles/12/docs/Areas/Desarrollo_Rural/Programa_Desarrollo_Rural _2007_2013/DOCUMENTO_COMPLETO_PDR_2007_2013.pdf

Programa de Desarrollo Rural de Canarias, 2007-2013. Gobierno de Canarias, 2008. Disponible en: http://www.pdrcanarias.org/index.php? option=com_content\&task= view\&id=105\&Itemid=128 (Fecha de consulta: 04/09/2012).

Programa de Desarrollo Rural de Castilla-La Mancha, 2007-2013. Junta de Comunidades de Castilla-La Mancha, 2008. Disponible en: http://www.jccm.es/web/index/ programa1212676998620pl/1212674430800.html (Fecha de consulta: 04/09/2012).

Programa de Desarrollo Rural de Castilla y León, 2007-2013. Junta de Castilla y León, 2008. Disponible en: http://www.jcyl.es/web/jcyl/AgriculturaGanaderia/es/Plantilla100/1185746059889/_/185746058890/Texto (Fecha de consulta: 04/09/2012).

Programa de Desarrollo Rural de Extremadura FEADER, 2007-2013. Junta de Extremadura, Consejería de Hacienda y Presupuesto, 2008. Disponible en: http://www. redr.es/recursos/doc/Politicas_rurales/Programa_desarrollo_rural/1165156936_21 12009174653.pdf y http://www.redr.es/recursos/doc/Politicas_rurales/ Programa_ desarrollo_rural/836976481_211200917485.pdf (Fecha de consulta: 04/09/2012).

Programa de Desarrollo Rural de la Comunidad de Madrid, 2007 - 2013. Comunidad de Madrid, Dirección general de agricultura y desarrollo rural, 2008. Disponible en: http://www.madrid.org/cs/Satellite?c=CM_Actuaciones_FA\&cid=1142583998613 \&idConsejeria $=1109266187260 \&$ \&idListConsj $=1109265444710$ \&language $=e s \& p$ agename $=$ ComunidadMadrid\%2FEstructura\&sm $=1109265843983$ (Fecha de consulta: 04/09/2012). 
Programa de Desarrollo Rural de la Comunitat Valenciana 2007-2013. Generalitat Valenciana, 2008. Disponible en: http://www.redr.es/recursos/doc/Politicas_rurales/Pro grama_desarrollo_rural/212837347_2112009175447.pdf (Fecha de consulta: 04/09/2012).

Programa de Desarrollo Rural de las Illes Balears 2007-2013. Govern de les Illes Balears, Conselleria d'agricultura i pesca, 2008. Disponible en: http://www.magrama. gob.es/es/desarrollo-rural/temas/programas-ue/pdr_baleares_20072013_tomo_1_mod_2011_scc_v0408111_tcm7-150650.pdf (Fecha de consulta: 04/09/2012).

Programa de Desarrollo Rural del País Vasco 2007-2013. Gobierno Vasco, Departamento de Agricultura, Pesca y Alimentación, 2008. Disponible en: http://www. redr.es/recursos/doc/Politicas_rurales/Programa_desarrollo_rural/1701425140_21 1200917579.pdf (Fecha de consulta: 04/09/2012).

Programa de Desarrollo Rural del Principado de Asturias 2007-2013. Principado de Asturias, Consejería de Medio Ambiente y Desarrollo Rural, 2008. Disponible en: https://sede.asturias.es/Asturias/descargas/PDF\%20DE\%20TEMAS/Europa/Pro grama_Desarrollo_Rural.2007_2013.pdf (Fecha de consulta: 04/09/2012).

Programa de Desarrollo Rural de la Región de Murcia 2007-2013 (FEADER). Región de Murcia, 2008. Disponible en: http://www.redr.es/recursos/doc/Politicas_rurales/ Programa_desarrollo_rural/2051168992_2112009172940.pdf y http://www.redr. es/recursos/doc/Politicas_rurales/Programa_desarrollo_rural/1182692181_211200 9173056.pdf (Fecha de consulta: 04/09/2012).

\section{RESUMEN}

La delimitación del ámbito rural ha sido, tradicionalmente, un tema de notable preocupación científica. Los contrastes entre el mundo urbano y rural, bien visibles en el paisaje y no menos elocuentes en las características sociales y económicas de ambos, han despertado el interés por definir un límite que no siempre ha sido fácilmente detectable. Unas veces, mediante criterios cuantitativos y, otras, cualitativos, incluso una mezcla de ambos, se han etiquetado territorios con denominaciones no siempre dicotómicas que han dado a entender más bien la gradación que existe entre la ciudad y lo rural profundo. Los organismos internacionales no han sido ajenos a esta preocupación y, consiguientemente, han propuesto metodologías para catalogar los espacios y las gentes que los habitan según criterios de ruralidad. A ello se une el interés de los Programas de Desarrollo Rural que en el marco de la Unión Europea se vienen aplicando en los últimos veinticinco años y que tienen en el denominado «enfoque territorial» uno de los pilares sustanciales; ello ha motivado un nuevo impulso en la indagación de este tema que tiene un indudable interés operativo.

PALABRAS Clave: ruralidad; delimitación rural; programas de desarrollo rural. 


\begin{abstract}
The delimitation of rural areas has traditionally been a topic of considerable scientific interest. The contrasts between urban and rural areas, which are easily distinguishable both in the landscape and in their social and economic characteristics, have raised the interest to define a limit that has not always been easily detectable. Quantitative and qualitative criteria, and even a mixture of both, have been used to label rural and urban territory, sometimes using terminology that shows more of the gradation between the two concepts rather than their dichotomy. International agencies have not been oblivious to this concern, and have proposed methodologies for cataloging these areas and the people in them according to criteria of rurality. Furthermore, the "territorial approach» is one of the bases of the Rural Development Programs that have been applied in the European Union over the last twenty five years, which has given a new impulse to the research in this field.
\end{abstract}

KEY WORDS: rurality; delimitation; rural development programs.

\title{
RÉSUMÉ
}

La délimitation du domaine rural a été traditionnellement un sujet remarquable d'étude scientifique. Les contrastes entre le monde urbain et le monde rural, facilement visibles dans le paysage et non moins évidents dans les caractéristiques sociales et économiques de chacun d'entre eux ont éveillé l'intérêt sur la définition d'une limite qui n'a pas toujours été facilement détectable. Avec des critères parfois quantitatifs, parfois qualitatifs, voire avec un mélange des deux, des territoires ont été étiquetés avec des dénominations pas forcément dichotomiques qui ont permis surtout de comprendre la gradation existante entre la ville et le milieu profondément rural. Les organisations internationales ont participé aussi de cette préoccupation, et ils ont donc proposé des méthodologies pour cataloguer les espaces et les populations qui les habitent selon des critères de ruralité. À cela s'ajoute l'intérêt des Programmes de développement rural appliqués les vingt-cinq dernières années dans le cadre de l'Union Européenne et dont un des éléments essentiels est «l'approche territoriale»; cela a donné lieu à un nouvel essor des recherches sur ce sujet, dont l'intérêt opérationnel est hors de question.

Mots CLÉs: ruralité; délimitation rurale; programmes de développement rural. 